\title{
Cosmological abundance of colored relics
}

\author{
Christian Gross, ${ }^{1,2}$ Andrea Mitridate, ${ }^{2,3}$ Michele Redi, ${ }^{4,5}$ Juri Smirnov, ${ }^{6}$ and Alessandro Strumia ${ }^{1}$ \\ ${ }^{1}$ Dipartimento di Fisica dell'Università di Pisa, 56127 Pisa, Italy \\ ${ }^{2}$ INFN, Sezione di Pisa, 56127 Pisa, Italy \\ ${ }^{3}$ Scuola Normale Superiore, 56126 Pisa, Italy \\ ${ }^{4}$ INFN sezione di Firenze, Via G. Sansone 1; I-59100 Sesto Fiorentino, Italy \\ ${ }^{5}$ Department of Physics and Astronomy, University of Florence, 50019 Florence, Italy \\ ${ }^{6} \mathrm{CP}^{3}$-Origins and DIAS, University of Southern Denmark, 5230 Odense, Denmark
}

(Received 12 October 2018; published 31 January 2019)

\begin{abstract}
The relic cosmological abundance of stable or long-lived, charge neutral, colored particles gets reduced by up to 4 orders of magnitude by annihilations that occur after QCD confinement. We compute the abundance and the cosmological bounds on relic gluinos. The same postconfinement effect strongly enhances coannihilations with a lighter dark matter particle, provided that their mass difference is below a few giga-electron volts. Charged colored particles (such as stops) can instead form baryons, which can be (quasi)stable in some models.
\end{abstract}

DOI: $10.1103 /$ PhysRevD.99.016024

\section{INTRODUCTION}

Extensions of the Standard Model (SM) sometimes predict (quasi)stable colored particles. We show that, due to nonperturbative QCD effects, their relic abundance is significantly lower than previously expected, correspondingly reducing the phenomenological constraints.

Weak-scale supersymmetry has been considered the most motivated extension of the SM, as it allows one to control quadratically divergent corrections to the Higgs mass keeping them naturally small; improves the prediction for the gauge couplings in $\mathrm{SU}(5)$ unification; and provides dark matter (DM) candidates. The plausibility of the naturalness goal is now endangered by the lack of any new physics in LEP [1] and LHC data [2]. Furthermore, the Higgs mass is larger than what was predicted by the minimal supersymmetric Standard Model with weak-scale sparticles.

Split supersymmetry $[3,4]$ (where the new supersymmetric fermions are much lighter than the new supersymmetric scalars) abandoned the naturalness goal, retaining the two other good features, allowing one to fit the Higgs mass [5,6], and relaxing the possible supersymmetric flavor problem caused by weak-scale sfermions. If sfermions are very heavy, the light gauginos can become long-lived, giving peculiar signatures at colliders and potential cosmological problems. These were explored in

Published by the American Physical Society under the terms of the Creative Commons Attribution 4.0 International license. Further distribution of this work must maintain attribution to the author(s) and the published article's title, journal citation, and DOI. Funded by SCOAP ${ }^{3}$.
[7], where the relic gluino abundance (before late gluino decay in neutralino and colored SM particles) was computed including perturbative gluino annihilations at $T \sim M_{3}$ and arguing that one can neglect nonperturbative effects arising after confinement at $T \sim \Lambda_{\mathrm{QCD}}$. Such effects reduce the relic gluino abundance by a few orders of magnitude [8], thereby weakening cosmological bounds.

The relevance of confinement effects has been estimated in [9] in the case of colored charged particles. Unlike in the case of the neutral gluino, QCD bound states of charged particles can be formed or broken by emitting or absorbing photons. We will consider the case of (quasi)stable stop $\tilde{t}$.

In Sec. II we compute the thermal relic abundance of (quasi)stable gluinos, and in Sec. III we reconsider the cosmological bounds and discuss the associated phenomenology. Conclusions are given in Sec. IV.

\section{RELIC GLUINOS}

We consider a Majorana fermion in the adjoint of SU(3). In supersymmetric models this is known as gluino and denoted as $\tilde{g}$. The gluino can be stable if it is the lightest supersymmetric particle. Otherwise it can decay via squark exchange into a quark, an antiquark, and a neutralino or chargino, or radiatively into a gluon and a neutralino, with quarks and squarks in the loop. The resulting lifetime is long if sfermions have a much heavier mass $m_{\text {SUSY }}[10,11]$,

$$
\tau_{\tilde{g}}=\frac{4 \mathrm{~s}}{N}\left(\frac{m_{\mathrm{SUSY}}}{10^{9} \mathrm{GeV}}\right)^{4}\left(\frac{\mathrm{TeV}}{M_{\tilde{g}}}\right)^{5},
$$

where $N$ is an order-one function [11]. A stable or long lived gluino is probed and constrained by cosmology. 


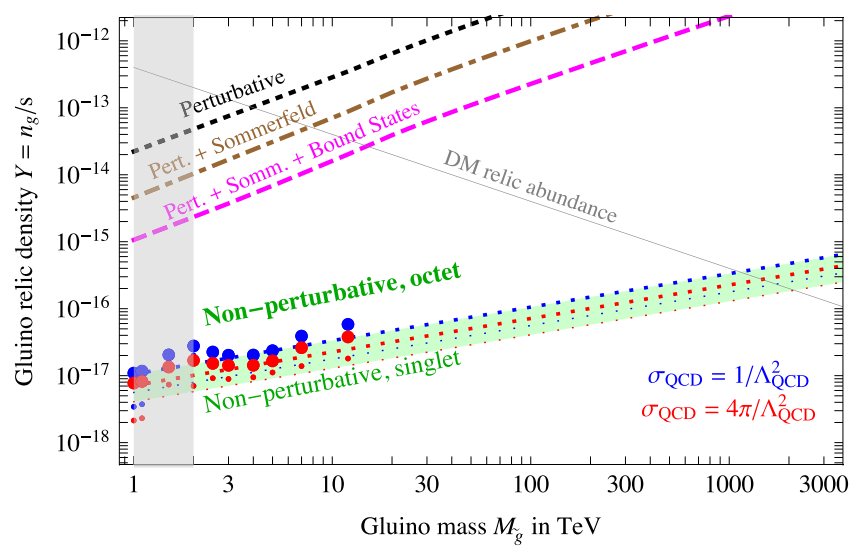

FIG. 1. Predicted gluino abundance. Relic stable gluinos exceed the DM density if $M_{\tilde{g}} \gtrsim \mathrm{PeV}$. The bands show the nonperturbative analytic result for $\sigma_{\mathrm{QCD}}=1 / \Lambda_{\mathrm{QCD}}^{2}$ (blue dots) and $\sigma_{\mathrm{QCD}}=4 \pi / \Lambda_{\mathrm{QCD}}^{2}$ (red dots). The thin (thick) lines assume that only singlet bound states (octet bound states, too) can form with QCD size; similarly, the small (large) dots show our numerical computation for some values of the gluino mass. The relations among the gluino mass, the gluino lifetime, and the scale of supersymmetry are displayed in Fig. 4.

\section{A. Computing the relic gluino abundance}

Figure 1 shows our result for the gluino relic abundance before their possible slow decays. This is computed as follows. The upper curves show the relic abundance after a first decoupling at $T \sim M_{\tilde{g}} / 25$, as computed in various approximations:

(1) at tree level in the perturbative expansion;

(2) taking into account Sommerfeld corrections the $s$-wave annihilation cross section is [Eq. (2.24) of [12], where the Sommerfeld $S$ factors are defined]

$$
\begin{aligned}
\sigma_{\mathrm{ann}} v_{\mathrm{rel}} & =\frac{27}{32} \sigma_{0}\left[\frac{1}{6} S_{3}+\frac{1}{3} S_{3 / 2}+\frac{1}{2} S_{-1}\right]+\frac{9}{8} \sigma_{0} S_{3 / 2}, \\
\sigma_{0} & =\frac{\pi \alpha_{3}^{2}}{M_{\tilde{g}}^{2}}
\end{aligned}
$$

(3) taking into account also a related effect: formation of bound states [13].

These effects reduce by about 1 order of magnitude the gluino abundance, controlled by the Boltzmann equation

$$
\frac{H z}{s} \frac{d Y_{\tilde{g}}}{d z}=-\left\langle\sigma_{\mathrm{ann}} v_{\mathrm{rel}}\right\rangle\left(Y_{\tilde{g}}^{2}-Y_{\tilde{g}}^{\mathrm{eq} 2}\right),
$$

where $z=M_{\tilde{g}} / T, Y_{\tilde{g}}=n_{\tilde{g}} / s, s$ is the entropy density at temperature $T$, and $H(T)$ is the Hubble constant.

If $\tau_{\tilde{g}}<M_{\mathrm{Pl}} / \Lambda_{\mathrm{QCD}}^{2} \sim \mu \mathrm{s}$, gluinos decay before the QCD phase transition leaving no cosmological effects. Otherwise, gluinos recouple as the temperature approaches the QCD scale, and their relic abundance is determined by a redecoupling at temperatures mildly below the QCD phase transition. At this point gluinos have formed $\tilde{g} g$ and/or $\tilde{g} q \bar{q}^{\prime}$ hadrons which scatter with large cross sections $\sigma_{\mathrm{QCD}}=$ $c / \Lambda_{\mathrm{QCD}}^{2}$ where $c \sim 1$, making about $M_{\mathrm{Pl}} / \Lambda_{\mathrm{QCD}} \sim 10^{19}$ scatterings in a Hubble time. For comparison, the proton-proton elastic scattering cross section at low energy is known to be $\sigma_{\mathrm{el}} \approx 100 \mathrm{mb}$, corresponding to $c \approx 23$.

Although gluinos are much rarer than gluons and quarks, occasionally, two gluino hadrons meet forming a $\tilde{g} \tilde{g}$ bound state. Classically such a state has angular momentum $\ell \approx$ $\mu v_{\text {rel }} b$ where $b \approx 1 / \Lambda_{\mathrm{QCD}}$ is the impact parameter; $\mu \simeq$ $M_{\tilde{g}} / 2$ is the reduced mass; and $v_{\text {rel }} \sim\left(T / M_{\tilde{g}}\right)^{1 / 2}$ is the relative velocity. Thereby $\ell \sim\left(M_{\tilde{g}} T\right)^{1 / 2} / \Lambda_{\mathrm{QCD}}$ is large for $M_{\tilde{g}} \gg \Lambda_{\mathrm{QCD}} \gtrsim T$. The quantum-mechanical total QCD cross section for forming $\tilde{g} \tilde{g}$ bound states is large because many partial waves contribute. This can be parametrized defining the maximal angular momentum as $\ell_{\max } \equiv$ $\sqrt{c / 2 \pi} M_{\tilde{g}} v_{\text {rel }} / \Lambda_{\mathrm{QCD}}$ obtaining (see, e.g., [14])

$\sigma_{\mathrm{QCD}}=\sum_{\ell=0}^{\ell_{\max }} \sigma_{\ell} \simeq \frac{c}{\Lambda_{\mathrm{QCD}}^{2}}, \quad \sigma_{\ell}=4 \pi \frac{2 \ell+1}{M_{\tilde{g}}^{2} v_{\mathrm{rel}}^{2}} \sin ^{2} \delta_{\ell}$,

where the phase shifts average to $\left\langle\sin ^{2} \delta_{\ell}\right\rangle \simeq 1 / 2$. This expectation is consistent with numerical results in toy calculable models [15].

The cross section relevant for reducing the gluino abundance is not $\sigma_{\mathrm{QCD}}$, but the smaller cross section $\sigma_{\text {ann }}$ for forming $\tilde{g} \tilde{g}$ states that annihilate into SM particles before being broken. Assuming that a $\tilde{g} \tilde{g}$ with angular momentum $\ell$ and energy $\sim T$ annihilates before being broken with probability $\wp_{\ell}(T)$, one has ${ }^{1}$

\footnotetext{
${ }^{1}$ This intuitive picture can be formally justified writing a network of Boltzmann equations, one for each bound state $I$ with different $\ell$ and $n$. Such equations contain the formation rates $\gamma_{I}$, the thermally averaged breaking rates $\Gamma_{I}^{\text {break }}$, the annihilation rates $\Gamma_{I}^{\mathrm{ann}}$, and the decay rates among the states $\Gamma_{I J}$. This is unpractical, given that hundreds of states play a relevant role. To get some understanding, we consider a toy system where only one state 1 can be produced, and only one state 3 can annihilate. State 1 can decay to state 2 , which can decay to state 3 . Then, assuming that the rates are faster than the Hubble rate, one can reduce the network of Boltzmann equations [13] to the single Boltzmann equation (3) for the total gluino density, controlled by an effective annihilation rate equal to $\wp \gamma_{1}$ where

$$
\begin{aligned}
\wp & =\mathrm{BR}_{12} \mathrm{BR}_{23}, \quad \mathrm{BR}_{12}=\frac{\Gamma_{12}}{\Gamma_{12}+\Gamma_{1}^{\text {break }}}, \\
\mathrm{BR}_{23} & =\frac{\Gamma_{23}}{\Gamma_{23}+\Gamma_{2}^{\text {break }}+\mathrm{BR}_{12} \Gamma_{1}^{\text {break }}},
\end{aligned}
$$

where the last term takes into account that 2 can upscatter to 1 . We see that $\wp$ does not depend on $\Gamma_{3}^{\text {ann }}$ and has the expected physical meaning. In view of QCD uncertainties we cannot compute all order unity factors, such that it is appropriate to employ the simpler intuitive picture.
} 


$$
\sigma_{\mathrm{ann}}=\sum_{\ell=0}^{\ell_{\max }} \sigma_{\ell} \wp_{\ell}
$$

A large cross section needs large $\ell$, but $\wp_{\ell}$ can be small at large $\ell$. We compute $\wp_{\ell}$ as the probability that the $\tilde{g} \tilde{g}$ bound state radiates an energy big enough to become unbreakable (bigger than $\approx T$ ) before the next collision, after a time $\Delta t \sim 1 / n_{\pi} v_{\pi} \sigma_{\mathrm{QCD}}$. In such a case it becomes unbreakable and keeps radiating until $\tilde{g} \tilde{g}$ annihilate.

The key quantity to be computed is thereby the power radiated by the relevant bound states that have $n, \ell \gg 1$. In the Abelian case, this is well approximated by its classical limit: Larmor radiation. Having assumed neutral constituents, we can neglect photon radiation. Similarly, gravitational radiation has cosmologically negligible rates $\Gamma_{\text {grav }} \sim E_{B}^{3} / M_{\mathrm{Pl}}^{2}$. The dominant radiation mechanism is gluon radiation, which differs from Abelian radiation because gluons are charged under QCD. This makes a difference when (as in our case) particles are accelerated because of the strong force itself. While a photon can be soft and its emission leaves the bound state roughly unchanged, an emitted gluon has its own QCD potential energy, and its emission changes the QCD potential among gluinos by an order one amount (in particular, a singlet bound state becomes an octet). As the classical limit of gluon emission is not known, we apply the quantum formulas.

We need to compute the power radiated by highly excited bound states, with sizes of order $1 / \Lambda_{\mathrm{QCD}}$. Smaller bound states can be approximated by the
Coulomb-like nonrelativistic limit of the QCD potential and can have various color configurations, in particular singlets and octets. At large distances, they appear as color singlets because they are surrounded by a soft gluon cloud at a distance of order $1 / \Lambda_{\mathrm{QCD}}$, which acts as a spectator when computing their inner behavior. In the opposite limit, states larger than $1 / \Lambda_{\mathrm{QCD}}$ can only be color-singlet hadrons. For our purpose what is needed are QCD-size bound states which are the most challenging, as confinement effects are starting to be relevant. We will estimate their effect into two opposite limits:

(8) assuming that color octet bound states are relevant, such that radiation is dominated by single-gluon emission (pion emission after hadronization) into singlet states. This is computed in Sec. II B.

(1) assuming that only color singlets exist, such that radiation is dominated by color-singlet double-gluon emission (pion emission after hadronization) among singlets. This is computed in Sec. II C.

While the two cases are analytically very different (e.g., different powers of the strong coupling), QCD is relatively strongly coupled so that the numerical final results in the two limiting cases will be similar.

Before starting the computations, we summarize generic results for QCD bound states.

\section{The bound states}

We compute the energy levels of the $\tilde{g} \tilde{g}$ bound states assuming the nonrelativistic QCD potential

$$
V(r)=\lambda\left\{\begin{array}{lll}
-\frac{\alpha_{3}(\bar{\mu})}{r}\left[1+\frac{\alpha_{3}}{4 \pi}\left(\frac{11}{7}+14\left(\gamma_{E}+\ln \bar{\mu} r\right)\right)\right] & r \ll 1 / \Lambda_{\mathrm{QCD}} \quad[16], \\
-\frac{\alpha_{3 \text { lattice }}}{r}+\sigma r & r \sim 1 / \Lambda_{\mathrm{QCD}} \quad[17],
\end{array}\right.
$$

where $\lambda=\left(C_{R}+C_{R^{\prime}}-C_{Q}\right) / 2$ for the potential among representation $R$ and $R^{\prime}$ in the $Q$ configuration with $C_{1}=0$, $C_{3}=4 / 3, C_{8}=3$ being the Casimirs. So $\lambda=3(3 / 2)$ for the potential among octets in the singlet (octet) configuration. Lattice simulations indicate $\alpha_{3 \text { lattice }} \approx 0.3$ and $\sigma \approx(0.4 \mathrm{GeV})^{2}$. The one-loop correction to the perturbative term means that the QCD potential is roughly given by the tree level potential with the strong coupling renormalized at the renormalization group equation scale $\bar{\mu} \approx 1 / r$. At finite temperature $\sigma(T) \approx$ $\sigma(0) \sqrt{1-T^{2} / T_{\mathrm{QCD}}^{2}}$ with $T_{\mathrm{QCD}} \approx 170 \mathrm{MeV}$ [17].

The product of two color octets decomposes as

$$
8 \otimes 8=1_{S} \oplus 8_{A} \oplus 8_{S} \oplus 10_{A} \oplus \overline{10}_{A} \oplus 27_{S},
$$

such that there are three attractive channels and the gluino bound states exist in the following configurations:

\begin{tabular}{c|cc|c}
\hline Color & $V$ i.e. & $\lambda$ & \multicolumn{2}{|c}{ Allowed $\ell$} \\
\hline $1_{S}$ & $-3 \alpha_{3} / r$ & 3 & even if $S=0, \quad$ odd if $S=1$ \\
$8_{A}$ & $-\frac{3}{2} \alpha_{3} / r$ & $3 / 2$ & even if $S=1, \quad$ odd if $S=0$ \\
$8_{S}$ & $-\frac{3}{2} \alpha_{3} / r$ & $3 / 2$ & even if $S=0, \quad$ odd if $S=1$
\end{tabular}.

The energy eigenvalues in a potential $V=-\alpha_{\text {eff }} / r+\sigma_{\text {eff }} r$ are [18]

$$
\begin{aligned}
E_{n \ell} & \approx \frac{\mu \alpha_{\mathrm{eff}}^{2}}{2}\left[-\frac{1}{n^{2} t}+12 \text { tnex }\right] \\
& \simeq \begin{cases}-\mu \alpha_{\mathrm{eff}}^{2} / 2 n^{2} & \text { Coulomb limit } \\
3\left(x \sigma_{\mathrm{eff}}\right)^{2 / 3} / 2 \mu^{1 / 3} & \text { string limit }\end{cases}
\end{aligned}
$$

where $\mu \approx M_{\tilde{g}} / 2$ is the reduced mass, $\ell=\{0,1, \ldots\}$ is the angular momentum, $n \geq 1+\ell, x=1.79(n-\ell)+\ell-0.42$, 


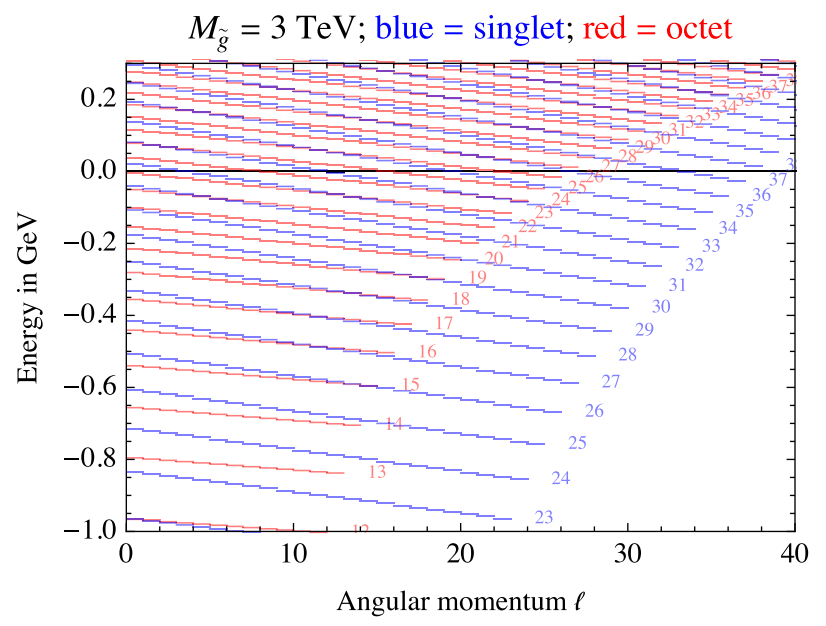

FIG. 2. Quantum energy levels of a $\tilde{g} \tilde{g}$ bound state which have energy close to 0 . Values of $n$ are shown.

$\varepsilon=\sigma_{\text {eff }} / 4 \alpha_{\text {eff }}^{3} \mu^{2}$ is a dimensionless number, and $t$ is the positive solution to $t=1-4 n^{3} \varepsilon x t^{3}$. In the limit where the Coulomb force dominates one has $t \simeq 1$ and $\varepsilon \simeq 0$; bound states have size $n^{2} a_{0}$ where $a_{0}=1 / \mu \alpha_{\text {eff }}$ is the Bohr radius. The linear force dominates when $n^{2} a_{0} \gg \sqrt{\alpha_{\text {eff }} / \sigma} \sim 1 / \Lambda_{\mathrm{QCD}}$.

Figure 2 shows the energy levels with nearly zero energy for $M_{\tilde{g}}=3 \mathrm{TeV}$.

\section{The breaking rate}

The probabilities $\wp_{\ell}$ that a given state radiates enough energy before being broken by a collision can be computed in two different ways.

Based on classical intuition, one can simply compare its energy loss rate with the breaking rate. While this simplification holds in the Abelian case, we have to deal with a non-Abelian dynamics, where gluon emission changes singlet to octet states, and vice versa. This is relevant, as singlet and octet decay rates are significantly different (especially for some singlet states that only decay through higher-order effects, as discussed below). It is not clear what is the classical limit of this system in the limit of large quantum numbers $n, \ell$.

We then perform a quantum computation, determining the $\wp_{\ell}$ by simulating transitions among the many different states. This is feasible up to masses $M_{\tilde{g}} \sim 10 \mathrm{TeV}$, because it involves a growing number of states at larger $M_{\tilde{g}}$.

We then need the breaking rate of the individual bound states. Thermal equilibrium between direct and inverse processes (also known as the Milne relation) does not allow one to infer the breaking rates from the total creation rate, because the latter is cumulative over all bound states. We assume that the breaking rate is given by the thermal average of the pion scattering cross section, assumed to be equal to $1 / \Lambda_{\mathrm{QCD}}^{2}$, and perform the thermal average $\left\langle\sigma_{\text {break }} v_{\text {rel }}\right\rangle$ over the distribution of pions with energies large enough to break the bound states. The number density of pions with enough energy to break a bound state with binding energy $E_{B}$ is

$n_{\pi}^{\mathrm{eq}}\left(E_{\pi}>E_{B_{I}}\right) \approx \frac{3\left(T\left(E_{B}+m_{\pi}\right)\right)^{3 / 2}}{2 \sqrt{2} \pi^{3 / 2}} \exp \left(-\frac{E_{B}+m_{\pi}}{T}\right)$,

such that $\left\langle\Gamma_{\text {break }}\right\rangle \approx\left\langle\sigma_{\text {break }} v_{\text {rel }}\right\rangle n_{\pi}^{\mathrm{eq}}\left(E_{\pi}>E_{B}\right)$.

\section{B. Color octet states and single-gluon emission}

We here assume that two colliding $\tilde{g}$ can form a $\tilde{g} \tilde{g}$ system with all 64 possible color configurations of Eq. (8), and with relative weights determined by combinatorics rather than by energetics. Then the effective annihilation cross section is determined summing over attractive channels as

$$
\sigma_{\mathrm{ann}} \propto \frac{1}{64} \sigma_{\mathrm{ann}}^{1}+\frac{1}{8}\left(\sigma_{\mathrm{ann}}^{8_{S}}+\sigma_{\mathrm{ann}}^{8_{A}}\right) .
$$

We fix the proportionality factor to $\approx 4$ such that the total cross section is $\sigma_{\mathrm{QCD}}=c / \Lambda_{\mathrm{QCD}}^{2}$, where $c \sim 1$ parametrizes our ignorance of the overall QCD cross section. The annihilation cross section is dominated by $\sigma_{\text {ann }}^{8_{A}}$ because the state $8_{A}$ radiates much more than 1 or $8_{S}$. Indeed, because of selection rules, single-gluon emission allows the following decays with $\Delta \ell= \pm 1$ :

$$
1 \rightarrow 8_{A}, \quad 8_{A} \rightarrow 1,8_{S} \quad 8_{S} \rightarrow 8_{A} .
$$

Taking hadronization into account two pions are emitted, such that the binding energy of the final state $E_{B}^{\prime}$ must be larger than $E_{B}+2 m_{\pi}$; otherwise, the decay is kinematically blocked. If the energy gap is somehow bigger than $\Lambda_{\mathrm{QCD}}$, inclusive decay rates can be reliably computed treating the gluon as a parton.

Since the 1 state is more attractive than $8_{S, A}$, the above conditions are easily satisfied for the $8_{A} \rightarrow 1$ decay, while $1 \rightarrow 8_{A}$ decays are kinematically blocked at larger $\ell$ and allowed at small enough $\ell$ (elliptic enough classical orbit), but suppressed with respect to the Abelian result.

In our numerical results we sum over all possible final states using wave functions computed in Wentzel-KramersBrillouin approximation using the Langer transformation. We also provide a simple approximated analytic result obtained assuming Coulombian wave functions (which is valid for deep final states, but not for the QCD-size initial states $)^{2}$

$\Gamma_{n \ell}\left(8_{A} \rightarrow 1_{S}\right) \approx \frac{2}{n^{2}} \alpha_{3}^{5} \mu, \quad W_{n \ell}\left(8_{A} \rightarrow 1_{S}\right)=\frac{8 \alpha_{3}^{7} \mu^{2}}{n^{3} \ell}$.

\footnotetext{
${ }^{2}$ In the same approximation, the smaller energy radiated into $8_{S}$ is given by a Larmor-like formula, given that the initial and the final states are equally attractive.
} 
The decay rate must be compared with the thermal breaking rate, which is given by pion scatterings such as $(\tilde{g} \tilde{g})+\pi \rightarrow(\tilde{g} g)+(\tilde{g} g)+\pi$. Since we considered bound states made of neutral gluinos, they are not broken by photon scatterings to leading order. The result is very simple: the $8_{A}$ decay rate is so fast that its actual value is irrelevant; all $8_{A}$ allowed states have $\wp_{\ell}=1$ at the relevant temperatures $T \lesssim \Lambda_{\mathrm{QCD}}$. On the other hand, $8_{S}$ and 1 states contribute negligibly. Then, the annihilation rate is controlled by a much simpler condition: $8_{A}$ bound states with binding energy $E_{B} \sim T$ only exist up to some maximal $\ell \leq \ell_{\max 8}$, which can be easily computed. For $M_{\tilde{g}}=3 \mathrm{TeV}$ Fig. 2 shows that $\ell_{\max 8} \approx 25$. For generic $M_{\tilde{g}} \gg T, \ell_{\max 8}$ is well approximated by imposing the vanishing of $E_{n \ell}$ in Eq. (10), finding

$$
\ell_{\max 8}=\left(12 \epsilon t^{2}\right)^{-1 / 4} \approx\left(\frac{3 M_{\tilde{g}}^{2} \alpha_{3}^{3}}{16 \sigma}\right)^{1 / 4}
$$

having approximated $t \approx 1$ in the last expression. Using Eq. (10), the deepest available singlet state has energy gap $\Delta E=\frac{9}{4} \sqrt{3 \alpha_{3} \sigma} \approx 0.9 \mathrm{GeV}$ (see also Fig. 2) and can only decay via higher order processes.

The effective annihilation cross section is

$$
\begin{aligned}
\sigma_{\mathrm{ann}} & \approx \frac{\sigma_{\mathrm{ann}}^{8_{A}}}{2} \approx \frac{1}{2} \sum_{\ell=0}^{\ell_{\mathrm{cr}}} \sigma_{\ell} \approx \frac{1}{2} \frac{2 \pi}{M_{\tilde{g}}^{2} v_{\mathrm{rel}}^{2}} \ell_{\mathrm{cr}}^{2}, \\
\ell_{\mathrm{cr}} & =\min \left(\ell_{\max }, \ell_{\max 8}\right) .
\end{aligned}
$$

At low (high) temperatures one has $\ell_{\text {cr }} \simeq \ell_{\text {max }} \propto v_{\text {rel }}$ $\left(\ell_{\text {cr }} \simeq \ell_{\max 8} \propto v_{\text {rel }}^{0}\right)$ such that the thermal average for $\ell \gg 1 \quad$ is $\quad\left\langle\sigma_{\text {ann }} v_{\text {rel }}\right\rangle \simeq 2 \sigma_{\mathrm{QCD}} \sqrt{T / \pi M_{\tilde{g}}} \quad\left(\left\langle\sigma_{\text {ann }} v_{\text {rel }}\right\rangle \simeq\right.$ $\sqrt{\left.3 \pi \alpha_{3}^{3} / 16 M_{\tilde{g}} T \sigma\right)}$. Taking the minimum of these two limits

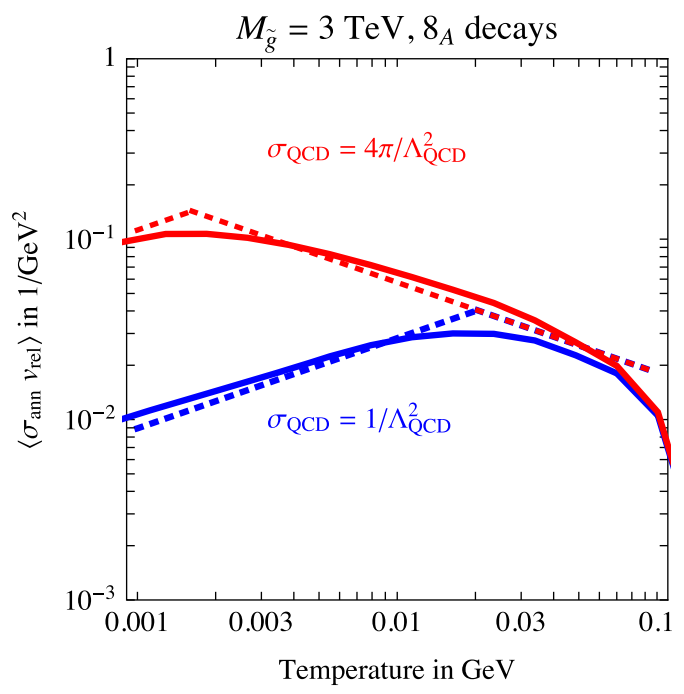

(which are equal at $T=T_{\mathrm{cr}}=\pi \sqrt{3 \alpha_{3}^{3} / \sigma} / 8 \sigma_{\mathrm{QCD}}$ with $\sigma_{\mathrm{QCD}}=c / \Lambda_{\mathrm{QCD}}^{2}$ ), we obtain an approximation valid at a generic intermediate $T$ :

$\left\langle\sigma_{\mathrm{ann}} v_{\mathrm{rel}}\right\rangle=\sigma_{\mathrm{QCD}} \sqrt{\frac{4 T}{\pi M_{\tilde{g}}}} \begin{cases}0 & \text { for } T>T_{\mathrm{QCD}}, \\ T_{\mathrm{cr}} / T & \text { for } T_{\mathrm{cr}}<T<T_{\mathrm{QCD}}, \\ 1 & \text { for } T<T_{\mathrm{cr}} .\end{cases}$

The Boltzmann equation of Eq. (3) is approximatively solved by

$$
\begin{aligned}
Y_{\tilde{g}}(\infty) & \approx \sqrt{\frac{45}{g_{\mathrm{SM}} \pi}} \frac{1}{M_{\tilde{g}} M_{\mathrm{Pl}}}\left[\int_{M_{\tilde{g}} / T_{\mathrm{QCD}}}^{\infty} d z \frac{\left\langle\sigma_{\mathrm{ann}} v_{\mathrm{rel}}\right\rangle}{z^{2}}\right]^{-1} \\
& \approx \frac{9 \sqrt{5 M_{\tilde{g}} / g_{\mathrm{SM}}}}{4 \sigma_{\mathrm{QCD}} T_{\mathrm{cr}}^{3 / 2} M_{\mathrm{Pl}}\left(3 \sqrt{T_{\mathrm{QCD}} / T_{\mathrm{cr}}}-2\right)}
\end{aligned}
$$

where the $d z$ integral is dominated by $T \sim T_{\mathrm{QCD}}$ : for $T_{\text {cr }} \ll$ $T_{\mathrm{QCD}}$ the abundance simplifies to

$$
\begin{aligned}
Y_{\tilde{g}}(\infty) & \approx \frac{1}{\pi M_{\mathrm{Pl}}} \sqrt{\frac{60 M_{\tilde{g}} \sigma}{g_{\mathrm{SM}} T_{\mathrm{QCD}} \alpha_{3}^{3}}} \\
& \approx 0.610^{-17} \sqrt{\frac{M_{\tilde{g}}}{3 \mathrm{TeV}} \frac{170 \mathrm{MeV}}{T_{\mathrm{QCD}}}} .
\end{aligned}
$$

The final relic abundance does not have a strong dependence on $\sigma_{\mathrm{QCD}}$, as it is only relevant at relatively low temperatures. The DM critical density is exceeded if $M_{\tilde{g}} \gtrsim \mathrm{PeV}$. Figure 3(a) shows the full numerical result

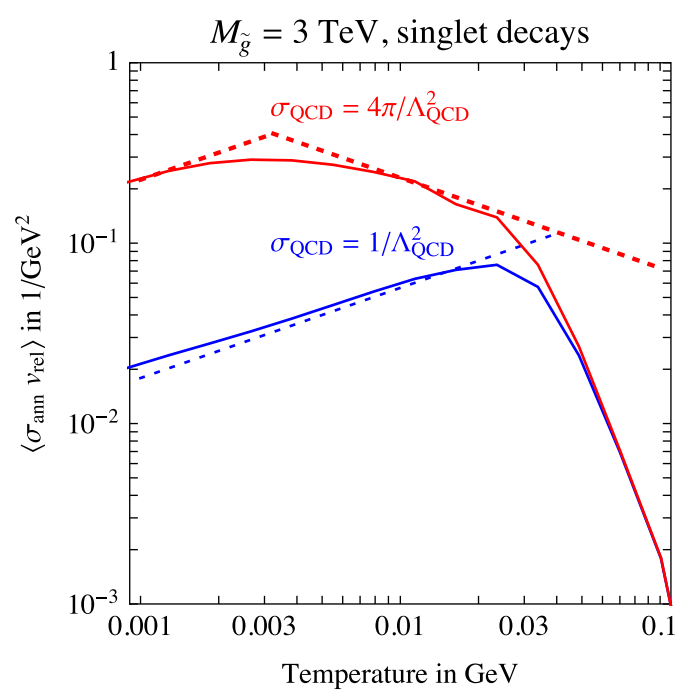

FIG. 3. The effective annihilation cross section of gluino $\tilde{g} \tilde{g}$ bound states, assuming that they form color-octet $8_{A}$ states (left) or only color-singlet states (right). The solid curves are the numerical computation, and the dashed lines are the maximal geometrical cross sections given by the analytic approximation. 
for $\left\langle\sigma_{\text {ann }} v_{\text {rel }}\right\rangle$, which agrees with the analytic maximal value (apart from some smoothing at $T \sim T_{\text {cr }}$ ) up to about $50 \mathrm{MeV}$ : thereby the numerical abundance is better reproduced lowering $T_{\mathrm{QCD}}$ to $50 \mathrm{MeV}$ in Eq. (19). This is done in the analytic estimate plotted in Fig. 1.

\section{Color-singlet states and two-gluon emission}

Single-gluon emission switches the color of the bound state as $1 \leftrightarrow 8$ and its angular momentum $\ell$ by \pm 1 : as a consequence kinematics blocks single-gluon decays of various color-singlet bound states, roughly all the ones in
Fig. 2 that do not have nearby octet states. In particular, decays of singlet states with maximal $\ell$ are blocked, and octet states with maximal $\ell$ can (but need not) decay to singlets with blocked decays.

We thereby take into account two-gluon emission, which allows for $1 \rightarrow 1$ decays with $\Delta \ell=\{0, \pm 2\}$. The rates of $2 g$ transitions are mildly suppressed by $\mathcal{O}\left(\alpha_{3}^{3}\right)$ compared with the $1 g$ decay rates. If the energy difference $\Delta E$ is much bigger than $\Lambda_{\mathrm{QCD}}$, gluon hadronization proceeds with unit probability and the $2 g$ decay widths can be computed using second order nonrelativistic perturbation theory [19],

$$
\begin{aligned}
\Gamma_{n, \ell \rightarrow n^{\prime}, \ell^{\prime}}^{2 g} \approx & \frac{3 \alpha_{3}^{2}}{16 \pi} \int_{0}^{\Delta E} d k k^{3}(\Delta E-k)^{3} \\
& \times\left.\sum_{m, m^{\prime}}\left|\left\langle\psi_{n, \ell, m}\left|r_{i}\left\{\frac{1}{-E_{n^{\prime}, \ell^{\prime}}+H_{8}-k}+\frac{1}{-E_{n^{\prime}, \ell^{\prime}}+H_{8}-(\Delta E-k)}\right\} r_{i}\right| \psi_{n^{\prime}, \ell^{\prime}, m^{\prime}}\right\rangle\right|\right|^{2},
\end{aligned}
$$

where $r_{i}=\{x, y, z\}$ is the relative distance between the two $\tilde{g} ; k$ is the momentum of the hadron produced in the hadronization of the two outgoing gluons, $\Delta E=E_{n^{\prime}, \ell^{\prime}}-E_{n, \ell}$ and $H_{8}$ the free Hamiltonian of the virtual intermediate octet state. The angular part of the matrix elements, already carried out in Eq. (20), imposes the selection rule $\left|\ell^{\prime}-\ell\right|=0,2$. The two-gluon $1 \leftrightarrow 1$ rates are given by an Abelian-like expression, unlike the one-gluon $1 \leftrightarrow 8$ transitions. The rates for $8 \rightarrow 8$ two-gluon transitions are given by a similar expression, with $H_{8}$ replaced by $H_{1}$.

Hadronization is possible down to the kinematical limit $\Delta E \approx 2 m_{\pi}$. However, the energy difference between two singlet states with maximal $\ell,|\Delta \ell|=2$, and nearby $n$ is $\sim \sigma^{3 / 4} \alpha_{3}^{-1 / 4} M_{\tilde{g}}^{-1 / 2}$, which, in view of the $M_{\tilde{g}}$ suppression, can be smaller than $2 m_{\pi}$. In such a case the decay can still proceed through off-shell pions, which produce photons and leptons. We estimate these suppressed decays following Sec. 5.6 of [20]. We neglect multigluon emission, which allows bigger jumps in $\ell$.

The $2 g$ rates are included in numerical computations which assume that QCD-scale color octets exist. The result was discussed in the previous subsection, as $2 g$ decays give a relatively minor correction.

We consider the opposite extreme possibility that octet states with QCD size do not exist and that only color singlets exist. We can again obtain an analytic lower bound on the final $\tilde{g}$ abundance by assuming that all singlet levels fall fast. Then the cross section $\sigma_{\mathrm{ann}} \approx \sigma_{\mathrm{ann}}^{1}$ is only limited by $\ell_{\max 1}=\sqrt{2} \ell_{\max 8}$ such that

$$
\left\langle\sigma_{\mathrm{ann}} v_{\mathrm{rel}}\right\rangle=\sigma_{\mathrm{QCD}} \sqrt{\frac{16 T}{\pi M_{\tilde{g}}}} \begin{cases}0 & \text { for } T>T_{\mathrm{QCD}}, \\ T_{\mathrm{cr}} / T & \text { for } T_{\mathrm{cr}}<T<T_{\mathrm{QCD}}, \\ 1 & \text { for } T<T_{\mathrm{cr}},\end{cases}
$$

where now $T_{\mathrm{cr}}=\pi \sqrt{3 \alpha_{3}^{3} / \sigma} / 4 \sigma_{\mathrm{QCD}}$. The resulting relic gluino abundance is 2 times lower than in Eq. (18) and with the new value of $T_{\mathrm{cr}}$. Figure 3(b) shows that this limit only holds at $T \lesssim 20 \mathrm{MeV}$, such that the analytic expression reproduces the numerical value for $Y_{\tilde{g}}$ by reducing $T_{\mathrm{QCD}}$ to $\sim 20 \mathrm{MeV}$.

\section{PHENOMENOLOGY}

\section{A. Cosmological bounds and signatures}

Bounds on quasistable relics depend on their lifetime $\tau_{\tilde{g}}$; on their mass $M_{\tilde{g}}$; on their relic abundance, which for gluinos we computed in terms of $M_{\tilde{g}}$; and on their decay modes. As mentioned above, we assume that gluinos decay to neutralinos [assumed to be the lightest supersymmetric particle (LSP)] plus either a gluon or a quark and an antiquark. Here we assume that half of the gluino energy is carried away by the LSP; if the LSP is not much lighter than the gluino, even less energy goes into SM states and one would obtain weaker bounds.

Our final result is plotted in Fig. 4, using the thick red dashed line of Fig. 1: even using updated experimental bounds (discussed below), our bounds on a (quasi)stable gluino are significantly weaker than those derived in [7]. The reason is that our relic density takes into account nonperturbative gluino annihilations and is much smaller than the "perturbative" gluino relic density assumed in [7]; see Fig. 1. In particular, we find that a (quasi)stable gluino just above the present collider bounds is still allowed provided that its lifetime is smaller than about $10^{12} \mathrm{~s}$ or larger than about $10^{22} \mathrm{~s}$.

In the rest of this section we summarize the various bounds on decaying relics plotted in Fig. 4, moving from smaller to larger lifetimes. 

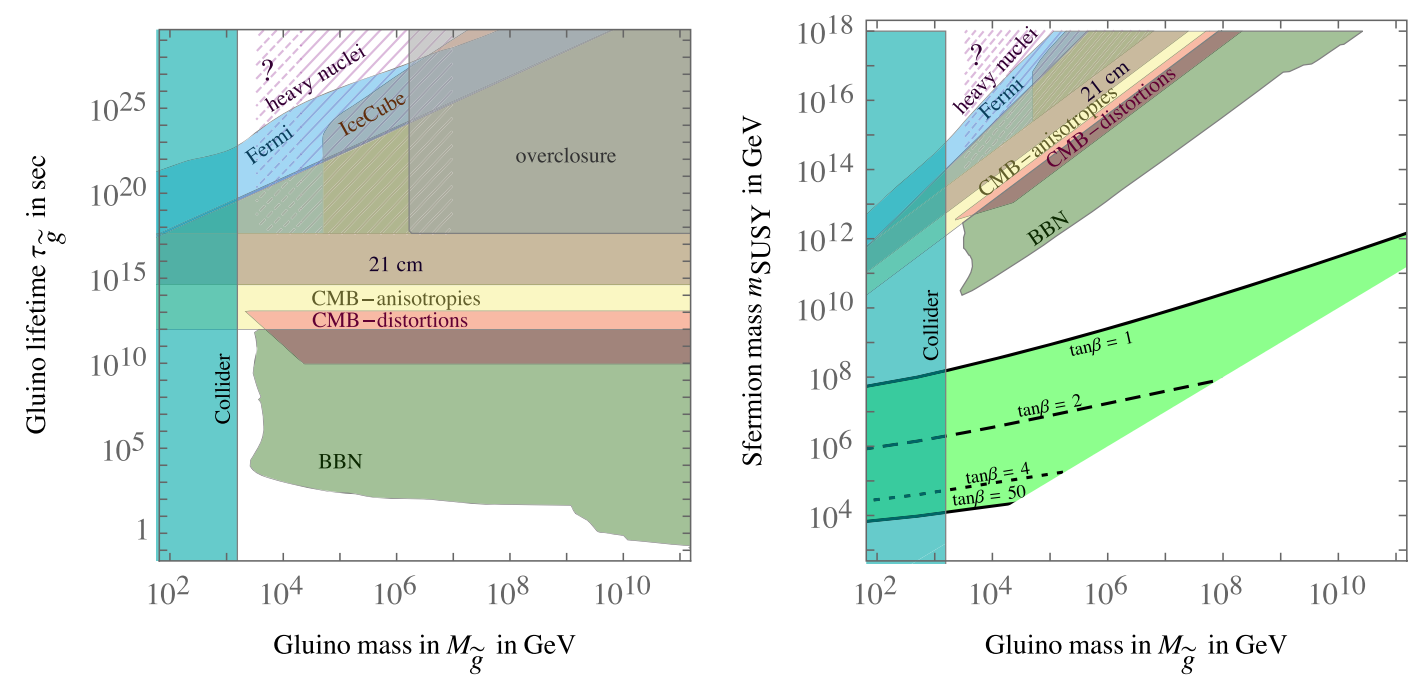

FIG. 4. Cosmological constraints on long-lived gluinos. Left: As a function of the gluino lifetime. Right: As a function of the sfermion mass scale $m_{\mathrm{SUSY}}$, which in split supersymmetry determines the gluino lifetime.

\section{Big bang nucleosynthesis}

A gluino that decays during big bang nucleosynthesis (BBN) can disturb the successful BBN predictions of light element abundances, which get affected in different ways, depending on the gluino lifetime (for more details see $[21,22])^{3}$ :

(i) For $0.1 \mathrm{~s} \lesssim \tau_{\tilde{g}} \lesssim 10^{2} \mathrm{~s}$ the nucleons and antinucleons, as well as the mesons with relatively long lifetimes of $\mathcal{O}\left(10^{-8}\right)$ s comparable to the typical mean free time for strong interactions (i.e., charged pions and kaons), which are produced by gluino decays, lose energy by electromagnetic interactions with the thermal bath before scattering with background nuclei. Thus they do not have enough energy to destroy light nuclei. However, they increase the interconversion rate of the protons and neutrons present in the thermal bath, e.g., via the strong interaction process $\pi^{-}+p \rightarrow \pi^{0}+n$. Since there are more $p$ than $n$ in the thermal bath, this effect increases the $n / p$ ratio and as a consequence the primordial ${ }^{4} \mathrm{He}$ mass fraction $Y_{p}$.

(ii) For $\tau_{\tilde{g}} \gtrsim 10^{2} \mathrm{~s}$ the gluino decay products do not thermalize before interacting with nuclei, due to the lower temperature of the plasma at these times. The still energetic nucleons (the mesons decay before they can interact) can thus hadrodissociate ${ }^{4} \mathrm{He}$, which in turn also increases the $\mathrm{D}$ abundance (e.g., via $p+{ }^{4} \mathrm{He} \rightarrow \mathrm{D}+{ }^{3} \mathrm{He}$ ).

\footnotetext{
${ }^{3}$ In addition, gluinos could also disturb the BBN predictions if they participate themselves in the nuclear reactions occurring during BBN $[23,24]$. This would be the case if the gluino $R$ hadrons bind into nuclei that are relevant during BBN. Since we do not know whether this is the case, we ignore such effects here.
}

(iii) For $\tau_{\tilde{g}} \gtrsim 10^{7} \mathrm{~s}$ photodissociation of ${ }^{4} \mathrm{He}$, which induces increased ${ }^{3} \mathrm{He}$ and $\mathrm{D}$ abundances, becomes relevant. Photodissociation is not relevant at earlier times because the $\gamma$ spectrum is cut off at the threshold energy $E_{\text {th }}^{\gamma} \approx m_{e}^{2} /(22 T)$ [25] for $e^{+} e^{-}$pair production from energetic $\gamma$ 's with thermal $\gamma$ 's, so that photons are not energetic enough to break up nuclei.

The resulting constraints have been computed in [21] and updated and improved in [22]. The constraints are given in the $\left(\tau_{X}, \xi_{X}\right)$ plane for different main decay modes of $X$, where $X$ is the unstable relic (the gluino in our case) and $\xi_{X}=E_{\mathrm{vis}} Y_{X}$ is its destructive power. Since we assume that half of gluinos' energy is carried away by the LSP we have $E_{\mathrm{vis}} \approx M_{\tilde{g}} / 2$. The bounds for the various hadronic decay modes are similar since in all cases they induce hadronic showers, and our bounds are based on the plot for the $t \bar{t}$ mode.

The effects from photodissociation depend only on the total injected energy, so that for $\tau_{\tilde{g}} \gtrsim 10^{7} \mathrm{~s}$ the bounds do not explicitly depend on $M_{\tilde{g}}$ to a good approximation. At earlier times, the effects depend on the number of hadrons produced in the hadronization process, which scales with a power of $M_{\tilde{g}}$. Thus we fit the bounds, given in [22] for $M_{X}=1 \mathrm{TeV}, 10 \mathrm{TeV}, 10^{2} \mathrm{TeV}, 10^{3} \mathrm{TeV}$, to a power-law function of $M_{\tilde{g}}$.

The left-handed panel of Fig. 4 shows the resulting bounds in green. In the right-hand panel we show the same bounds with the gluino lifetime computed as a function of the SUSY breaking scale $m_{\text {SUSY }}$.

\section{Distortion of the CMB blackbody spectrum}

Gluinos with lifetimes between $\sim 10^{7} \mathrm{~s}$ and $\sim 10^{13} \mathrm{~s}$ (the latter corresponds to recombination) can lead to deviations 
of the cosmic microwave background (CMB) spectrum from a blackbody form. When the Universe is $10^{7} \mathrm{~s}$ old, photon number changing processes such as double Compton scattering are not efficient any more, so that photons injected into the plasma can induce a chemical potential $\mu \simeq 1.41 \delta \epsilon / \epsilon$ [26] in the Bose-Einstein distribution of the CMB radiation, where [27]

$\frac{\delta \epsilon}{\epsilon} \simeq 4 \times 10^{-3} \sqrt{\frac{\tau_{\tilde{g}}}{10^{6} \mathrm{~s}}} \frac{M_{\tilde{g}} Y_{\tilde{g}} B_{\gamma}}{10^{-9} \mathrm{GeV}} \exp \left[-\left(\frac{6.1 \times 10^{6} \mathrm{~s}}{\tau_{\tilde{g}}}\right)^{5 / 4}\right]$.

After $\sim 4 \times 10^{11} \Omega_{b} h^{2}$ s [27], elastic Compton scatterings do not maintain thermal equilibrium anymore. An injection of photons "Comptonizes" the spectrum; i.e., it leads to a mixture of blackbody spectra of different temperatures. This is described by the Compton $y$ parameter, given by $y=\delta \epsilon / 4 \epsilon[26]$.

The 95\% C.L. limits from the FIRAS instrument on the COBE satellite are $|\mu|<9 \times 10^{-5}$ and $|y|<1.5 \times 10^{-5}$ $[28,29]$. The resulting constraints on the gluino lifetime are shown in pink in Fig. 4. Here we assumed that $\sim 45 \%$ (see, e.g., [30]) of the energy that is not carried away by the LSP goes into photons. The resulting bounds are less constraining than the BBN bounds. However, future bounds from PIXIE [31] will be stronger by 2 to 3 orders of magnitude, making the $\mathrm{CMB}$ sensitivity potentially stronger than the BBN constraints.

\section{CMB anisotropies}

The electromagnetic energy ejected into the gas at or after recombination by decaying relics modifies the fraction of free electrons and heats the intergalactic medium. This leads to modifications of the CMB angular power spectrum, measured by Planck. The maximally allowed density of a long-lived relic as a function of its lifetime has been computed assuming decay products with fixed energies in the range from $10 \mathrm{keV}$ up to $10 \mathrm{TeV}$ [32], respectively, $1 \mathrm{TeV}$ [33]. The $e^{+}, e^{-}, \gamma$ from hadronic decays do not have fixed energies, and moreover we do not know the energy spectrum of the decay products of relics with a mass significantly larger than $10 \mathrm{TeV}$. For very large gluino masses the bounds we show are therefore only indicative. We consider the middle of the band in [33] and obtain bounds by assuming that half of gluinos' energy goes into SM states and that $60 \%$ (see, e.g., [30]) of the latter goes into $e^{+}, e^{-}, \gamma$. In Fig. 4 we show the resulting constraints for a gluino with a lifetime $\gtrsim 10^{12} \mathrm{~s}$ in yellow.

\section{The $21 \mathrm{~cm}$ line}

If confirmed, the observation of an absorption feature in the low energy tail of the CMB spectrum [34] allows us to put an upper bound on the temperature of the intergalactic medium (IGM) at redshift $z \approx 17$. Decays of relic particles during the dark ages are constrained, mainly because they inject energy in the IGM heating it, erasing the absorption feature. Bounds on decaying DM particles, with masses up to $10 \mathrm{TeV}$, have been computed in [35-37]. We rescale these bounds to a generic abundance, still assuming that half of gluino energy goes into SM states and that $60 \%$ (see, e.g., [30]) of the latter goes into $e^{+}, e^{-}, \gamma$. The result is shown in Fig. 4. Similar to the case of the CMB bounds in the previous section, the $21 \mathrm{~cm}$ bounds for very large gluino masses are only indicative and subject to significant uncertainty.

\section{Constraints from gamma-ray telescopes and neutrino detectors}

Decaying gluinos with larger lifetimes are constrained by the measurement of cosmic ray spectra, in particular of photons or neutrinos. We adopt the results of the authors of [38] who computed limits on the lifetime of DM decaying to $b \bar{b}$, from data from the Fermi gamma ray telescope and the neutrino detector IceCube, up to a DM mass of $10^{12} \mathrm{GeV}$. We rescale the bounds of [38] taking into account that the density of our relics differs from the DM density. The authors of Ref. [38] derive bounds assuming a relic that decays to $b \bar{b}$. We assume that $50 \%$ of the gluino's energy goes to the LSP and the rest goes into hadronic decay channels, which lead to similar spectra as $b \bar{b}$. Figure 4 shows the resulting constraints on a long-lived gluino from Fermi (in blue) and IceCube (in orange). The IceCube limits exceed the bounds from Fermi data for $M_{\tilde{g}} \gtrsim 10^{7} \mathrm{GeV}$.

\section{Searches for supermassive nuclei}

Coming finally to stable gluinos, lattice simulations indicate that they would form neutral $\tilde{g} g$ hadrons [39], as well as a minor component of baryonic states such as $\tilde{g} u u d$ (according to [40] the lightest gluino baryon could be $\tilde{g} u d s$ ). They behave as strongly interacting Dark Matter. This is allowed by the bounds from direct detection experiments performed in the upper atmosphere and by searches for supermassive nuclei in the Earth and in meteorites if their relic abundance is a few orders of magnitude smaller than the cosmological DM abundance, although the precise bound is subject to considerable uncertainties (see the discussion in [8]). In Fig. 4 we indicate the tentative constraints that arise from the search for supermassive nuclei in meteorites by Rutherford backscattering of ${ }^{238} \mathrm{U}, N_{\text {SIMP }} /\left.N_{n}\right|_{\text {meteorites }} \lesssim 2 \times$ $10^{-12}$ [41], assuming a heavy nuclei capture cross section of $\sigma_{\text {capture }}=10^{-2} / \Lambda_{\mathrm{QCD}}^{2}$. Presumably, there is still an open window, from $\mathrm{TeV}$ masses above the LHC [9] up to about $10 \mathrm{TeV}$.

\section{Higgs mass}

In the right panel of Fig. 4 we considered split supersymmetry, such that the gluino lifetime is computed as a function of the sfermion mass $m_{\text {SUSY }}$; see Eq. (1). 
This scale is further constrained within the split minimal supersymmetric Standard Model by the observed Higgs mass, which is reproduced within the green region (for different values of $\tan \beta)$ in the $\left(M_{3}, m_{\mathrm{SUSY}}\right)$ plane. We computed $M_{h}$ as in [6], assuming that gauginos and Higgsinos are degenerate at the gluino mass $M_{3}$ and that all scalars are degenerate at $m_{\text {SUSY }}$. Allowing the masses to vary and taking into account uncertainties on $M_{t}$ and $\alpha_{3}$ slightly expands the region. Within the Higgs-allowed region the gluino decays promptly on cosmological timescales, evading all cosmological bounds.

No prediction for the Higgs mass arises in extensions of the minimal supersymmetric Standard Model. However, roughly the same region is obtained imposing the metastability bound on Higgs vacuum decay, which implies that the Higgs quartic $\lambda_{H}$ cannot be too negative, $\lambda_{H} \gtrsim-0.05$. A substantially larger $m_{\text {SUSY }}$, such that the gluino is long lived, is obtained assuming that Higgsinos are heavy (possibly with masses of order $m_{\mathrm{SUSY}}$ : in such a case the renormalization group equation for the Higgs quartic are those of the SM (with slightly different values of $g_{2,3}$ due to the light gluino and wino), and the Higgs quartic can remain positive up to $m_{\mathrm{SUSY}} \sim M_{\mathrm{Pl}}$ within the uncertainty range for the top quark mass.

\section{B. Collider signals}

Next, we discuss some aspects of the phenomenology of long-lived gluinos at hadron colliders, in particular LHC. Long-lived gluinos can be pair produced and after hadronization form long-lived hybrid states with SM quarks and gluons, known as " $R$ hadrons." We conservatively assume that the signal at the LHC is just energy deposit in the calorimeter, rather than charged particles in the tracker. It is difficult to trigger on these events and so an initial state jet is required. The LHC places the limit $M_{\tilde{g}}>1.55 \mathrm{TeV}$ on a Majorana gluino [42].

The other possibility is the production of a $\tilde{g} \tilde{g}$ bound state. Assuming that states with $\ell=0$ dominate the rates, they are color $8_{A}$ with spin $S=1$ and color singlets or $8_{S}$ with $S=0$ [see Eq. (9)]. The production cross sections are given by gluon and quark fusion, respectively,

$$
\begin{aligned}
\sigma_{0} & =\sum_{n=1}^{\infty} \frac{\mathcal{L}_{g g}}{2 M_{\tilde{g}} s n^{3}}\left(\Gamma_{g g}^{1}+8 \Gamma_{g g}^{8_{S}}\right) \\
& =\frac{\zeta(3) \mathcal{L}_{g g}}{2 M_{\tilde{g}} s}\left(\Gamma_{g g}^{1}+8 \Gamma_{g g}^{8_{S}}\right), \\
\sigma_{1} & =2 \sum_{n=1}^{\infty} \frac{\mathcal{L}_{u u} \Gamma_{u u}^{8_{A}}+\mathcal{L}_{d d} \Gamma_{d d}^{8_{A}}}{M_{\tilde{g}} s n^{3}} \\
& =\frac{2 \zeta(3)}{M_{\tilde{g}} s}\left(\mathcal{L}_{u u} \Gamma_{u u}^{8_{A}}+\mathcal{L}_{d d} \Gamma_{d d}^{8_{A}}\right),
\end{aligned}
$$

where $\mathcal{L}_{i j}$ is the luminosity of partons $i j$. The decay rates are given by [8]

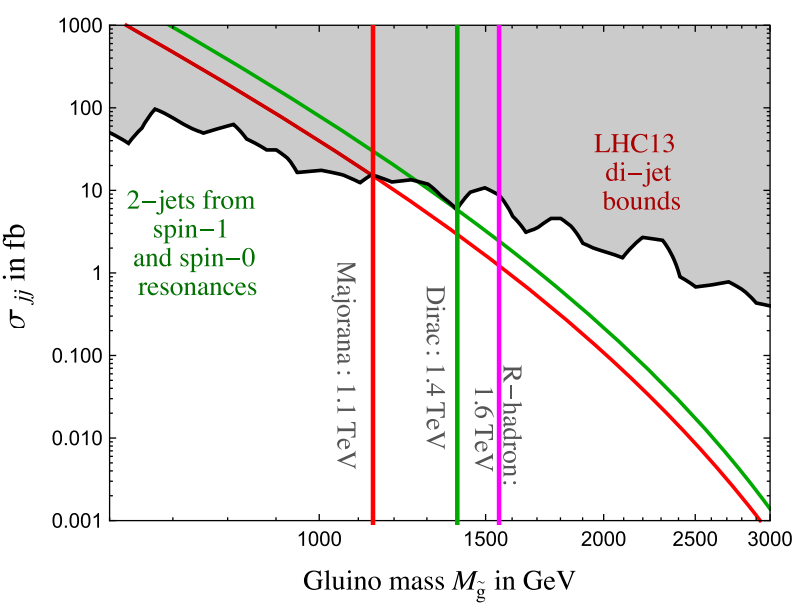

FIG. 5. The black curve is the dijet upper bound on the cross section for production of spin-1 and spin-0 bound states from LHC data at $13 \mathrm{TeV}$; the red (green) curve is the theoretical prediction assuming a Majorana (Dirac) gluino. From this we derive the experimental bounds (vertical lines). The thin vertical line shows the bound from $R$-hadron searches.

$\frac{\Gamma_{g g}^{1}}{M_{\tilde{g}}}=\frac{9 \alpha_{3}^{5} \lambda_{1}^{3}}{2 F}, \quad \frac{\Gamma_{g g}^{8_{S}}}{M_{\tilde{g}}}=\frac{9 \alpha_{3}^{5} \lambda_{8}^{3}}{8 F}, \quad \frac{\Gamma_{q q}^{8_{A}}}{M_{\tilde{g}}}=\frac{3 \alpha_{3}^{5} \lambda_{8}^{3}}{2 F}$,

with $F=2$ for the Majorana gluino and $F=1$ for a Dirac particle, and with the channel strengths $\lambda_{1}=3$ and $\lambda_{8}=3 / 2$.

Since the resonances annihilate to two gluons or two quarks, we assume a $100 \%$ branching ratio to two jets and apply the LHC dijet bounds [43] to the sum of the cross sections. In Fig. 5 we compare the bounds on the resonances to, slightly stronger, the $R$-hadron bound.

Concerning future colliders, the expected reach of a $100 \mathrm{TeV}$ hadron collider with $1000 \mathrm{fb}^{-1}$ is 7 (9) $\mathrm{TeV}$ for a Majorana (Dirac) gluino, having used [44] to perform an approximate rescaling. The $R$-hadron search would then reach $10 \mathrm{TeV}$ and $14.5 \mathrm{TeV}$, respectively. Thus a $100 \mathrm{TeV}$ collider would reach the benchmark mass of a thermally produced Dirac gluino, which recently was found to be a dark matter candidate [8].

\section{Implications for dark matter coannihilations}

The thermal relic abundance of a particle is affected by coannihilations with particles of similar mass. One example is coannihilations of neutralino DM with heavier colored particles, e.g., gluinos. Coannihilations can be enhanced by Sommerfeld corrections [12] and bound-state formation $[13,45]$. We point out that a much bigger effect is produced by nonperturbative QCD effects after the QCD phase transition, if the mass splitting $\Delta M$ between the coannihilating species is comparable to or smaller than $\Lambda_{\mathrm{QCD}}$. Such a near degeneracy is unnatural. This is shown in Fig. 7(a) in the neutralino/gluino coannihilation case, assuming that squarks mediate fast neutralino/gluino rates. 
We see that the neutralino mass that reproduces the observed DM density gets much higher at $\Delta M \lesssim \mathrm{GeV}$. In the limit $\Delta M \ll \mathrm{GeV}$ the relic abundance is dominantly set by the new QCD annihilations. As a result, the neutralino mass can reach up to a PeV, heavier than the maximal relic DM mass allowed if DM annihilations are dominated by partial waves with low $\ell$ [14].

\section{Quasistable squark}

In the previous sections we considered a Majorana gluino. A real scalar in the octet of $\mathrm{SU}(3)_{c}$ would behave similarly to the Majorana gluino. On the other hand, a (quasi)stable particle in the fundamental 3 of color $\mathrm{SU}(3)_{c}$ can behave in a qualitatively different way. Since the 3 is a complex representation, the particle must be a complex scalar or a Dirac fermion, which can carry a conserved charge.

For definiteness, we consider the possibility of a (quasi) stable squark, and more specifically a stop $\tilde{t}$, as renormalization group equation effects tend to make $\tilde{t}$ lighter than other squarks. A stable stop arises if $\tilde{t}$ is the lightest SUSY particle (LSP) and $R$ parity is conserved. A quasistable stop arises if $R$ parity is almost conserved, or if the stop decays slowly into the LSP: this can happen, e.g., when the LSP is a gravitino. Collider bounds on stops [46] tend to ignore the possibility that the lighter stop $\tilde{t}$ is the (quasi)stable LSP, because it is perceived to be already excluded by cosmology.

In cosmology, perturbative QCD $\tilde{t}^{*} \rightarrow g g$ annihilations dominate over $\tilde{t} \tilde{t} \rightarrow t t$ annihilations and leave a roughly equal amount of relic $\tilde{t}$ and $\tilde{t}^{*}$. Perturbative QCD annihilations are enhanced by Sommerfeld and bound-state effects, computed in [13]. The relic $\tilde{t}$ abundance after perturbative annihilations is plotted in Fig. 6 and approximated by

$$
\frac{n_{\tilde{t}}}{s} \approx \frac{M_{\tilde{t}}}{M_{\mathrm{Pl}} \alpha_{3}^{2}}
$$

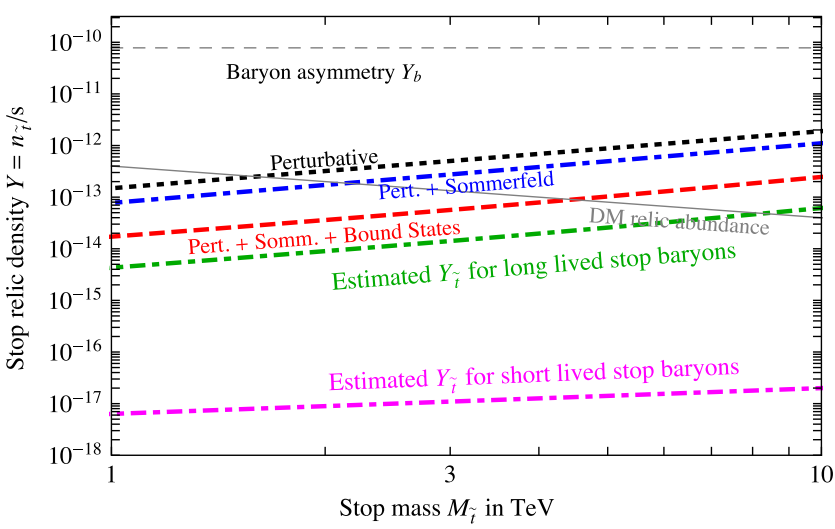

FIG. 6. Stop relic abundances. The $\tilde{t} \tilde{t} \tilde{t}$ baryons could be relatively long lived and have an abundance not suppressed by QCD confinement effects.
For $M_{\tilde{t}}<\mathrm{PeV}$ this is smaller than the baryon asymmetry $n_{b} / s \sim 10^{-10}$ that we neglect given that its effect is model dependent. Indeed, we do not know how the baryon asymmetry is generated: it might be generated at the weak scale such that it would not affect heavier stops. Even if a baryon asymmetry is present at stop decoupling, $\tilde{t} \bar{t} \leftrightarrow \tilde{t}^{*} t$ scatterings could easily concentrate the baryon asymmetry to lighter baryons fast enough that the asymmetry is irrelevant for stops. If instead the baryon asymmetry enhances the relic stop abundance, bounds would become stronger.

After the QCD phase transition, stops form hadrons. In view of the large QCD cross sections, the stop hadrons with dominant abundance are deeply bounded states that contain stops only. They are $\tilde{t}^{*}$ and the charged baryons $\tilde{t} \tilde{t} \tilde{t}$. Both fall to the ground state and decay through annihilations of the constituents. In particular, a bound state containing two or more stops decays, in its ground state, with a lifetime $\Gamma_{\tilde{t} \tilde{t}} \sim \alpha_{3}^{3} M_{\tilde{t}}^{3} \sigma_{\tilde{t} \tilde{t}} v_{\text {rel }}$ where the cross section for $\tilde{t} \tilde{t} \rightarrow t t$ can be roughly estimated as $\sigma_{\bar{t} \bar{t}} v_{\text {rel }} \sim \sum_{i=\{1,2,3\}} \alpha_{i}^{2} / M_{i}^{2}$, ignoring possible extra velocity suppressions. Then, $\Gamma_{\tilde{t} \bar{t}}$ is cosmologically fast unless gauginos (with masses $M_{i}$ ) are heavier than $\sim 10^{10} \mathrm{GeV}$.

We expect a roughly equal number of $\tilde{t}^{*}$ annihilations for each produced $\tilde{t} \tilde{t} \tilde{t}$ given that QCD group algebra implies that both $\tilde{t}^{*}$ and $\tilde{t} \tilde{t}$ feel an attractive Coulombian QCD force, such that they can form deep, unbreakable, Coulombian bound states. Assuming that a $\tilde{t}$ binds with probability $\wp$ to a $\tilde{t}$ and with probability $1-\wp$ to a $\tilde{t}^{*}$ and thereby that a deep $\tilde{t} \tilde{t}$ binds with probability $1-\wp$ to $\tilde{t}$ and with probability $\wp$ to a $\tilde{t}^{*}$, the average number of $\tilde{t} \tilde{t}^{*}$ per produced baryon is

$$
\frac{\left\langle N_{\tilde{t}^{*}}\right\rangle}{\left\langle N_{\tilde{t} \tilde{t} \tilde{t}}+N_{\tilde{t}^{*} \tilde{t}^{*} \tilde{t}^{*}}\right\rangle}=\frac{1 / \wp+1 /(1-\wp)-1}{r+1 / r-1} .
$$

This equals 3 assuming no baryon asymmetry $r \equiv N_{\tilde{t}} / N_{\tilde{t}}^{*}$ and $\wp=1 / 2$, namely neglecting that $\tilde{t}^{*}$ is more attractive than $\tilde{t} \tilde{t}$. Extra hadrons and mesons that contain quarks have a much smaller abundance that is not relevant here. If the charge 2 states $\tilde{t} \tilde{t} \tilde{t}$ decay fast on cosmological scales, final abundances and bounds are similar to the gluino case. If (quasi)stable, they are instead subject to strong cosmological constraints. In particular, during $\mathrm{BBN} \tilde{t}^{*} \tilde{t}^{*} \tilde{t}^{*}$ can bind to ${ }^{4} \mathrm{He}$ reducing its charge and thereby the Coulomb suppression of nuclear reactions, opening up a new channel for ${ }^{6} \mathrm{Li}$ production,

$$
\left(\tilde{t}^{*} \tilde{t}^{*} \tilde{t}^{*}{ }^{4} \mathrm{He}\right)+D \rightarrow{ }^{6} \mathrm{Li}+\tilde{t}^{*} \tilde{t}^{*} \tilde{t}^{*},
$$

which can strongly alter lithium abundances (see [47] for a brief review). Charge -1 states with lifetime $\gtrsim 10^{5}$ are subject to the BBN bound $Y \lesssim 2.5 \times 10^{-17}$ [23]. A study of analogous constraints on relics with charge -2 is beyond the scope of this paper. 

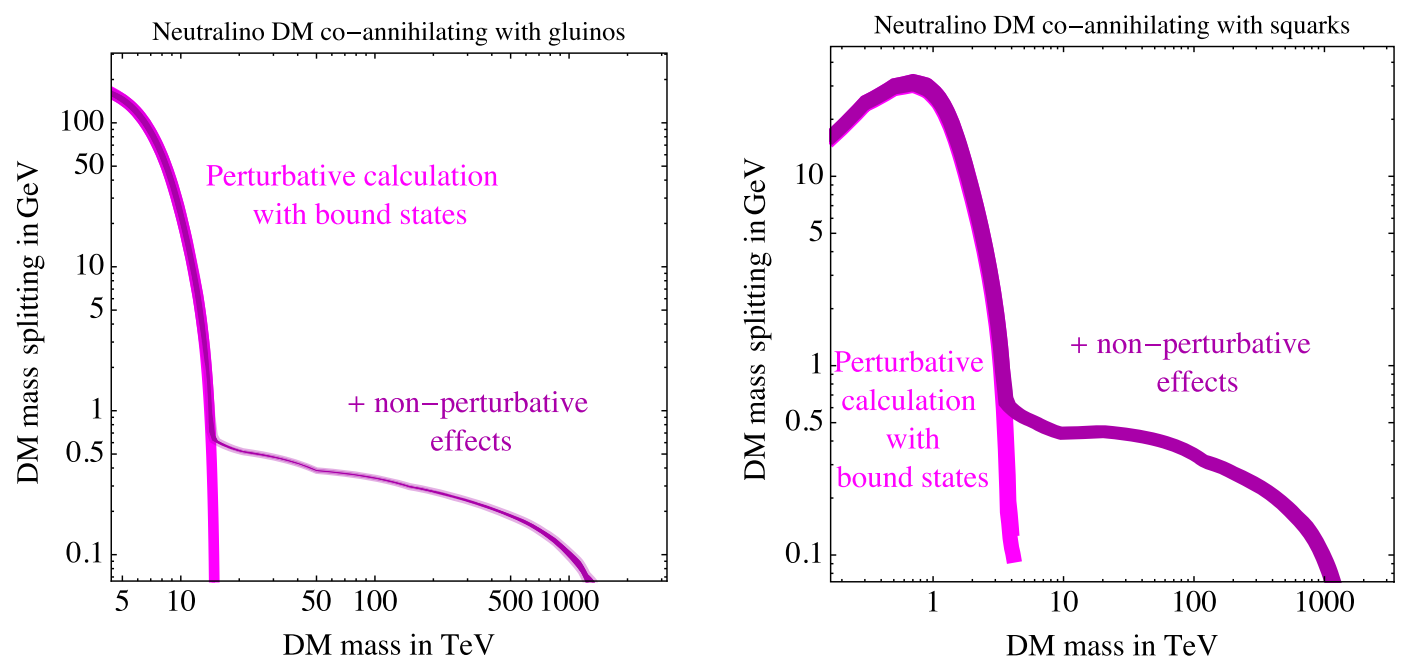

FIG. 7. Nonperturbative QCD annihilations that take place at $T \lesssim \Lambda_{\mathrm{QCD}}$ significantly increase the DM neutralino mass such that the observed DM abundance is reproduced through coannihilations with gluinos (left) or stops (right), if their mass difference with neutralinos is smaller than a few GeV. In the case of stops (right panel), the big effect is only estimated and present only if stop baryons decay to SM particles before decaying to neutralinos; otherwise confinement only gives a $\mathcal{O}(1)$ effect.

Next, we study the scenario where a quasistable stop coannihilates with a slightly lighter DM neutralino. Postconfinement effects are relevant if $\Delta M \lesssim \mathrm{GeV}$. Roughly half of the stops form $\tilde{t}^{*}$ mesons, and the others form $\tilde{t} \tilde{t} \tilde{t}$ baryons. The impact on the $\mathrm{DM}$ abundance is very different, depending on which process dominates $\tilde{t} \tilde{t} \tilde{t}$ decays. If it is dominated by stop annihilations into SM particles, postconfinement effects strongly suppress the DM abundance, similar to the gluino/neutralino coannihilation scenario. A much smaller order one effect is obtained if instead stops decay to DM neutralinos and SM particles with rate $\Gamma_{\tilde{t}} \gtrsim \Gamma_{\tilde{t} \tilde{t}}$. The region where the DM abundance is reproduced is estimated in Fig. 7(b) in the two extreme possibilities, having assumed $\sigma_{\mathrm{QCD}}=1 / \Lambda_{\mathrm{QCD}}^{2}$.

\section{CONCLUSIONS}

We have reconsidered the relic abundance of neutral colored relics, finding that hadron collisions at temperatures below the QCD scale reduce it by a few orders of magnitude. In particular, we considered a quasistable gluino: Fig. 1 shows its relic abundance, and Fig. 4 the cosmological constraints, taking into account the new effect and new data.

Coannihilations between gluinos and neutralino DM are similarly strongly affected by confinement, provided that their mass difference is smaller than a few $\mathrm{GeV}$, as shown in Fig. 7(a).

In Sec. III D we considered charged colored relics, considering, in particular, the case of a quasistable stop. In this case, confinement gives a big contribution to coannihilations with neutralinos only if $\tilde{t} \tilde{t} \tilde{t}$ baryons decay into SM particles via $\tilde{t} \tilde{t} \rightarrow t t$ before that stop decays to neutralinos.

\section{ACKNOWLEDGMENTS}

C. G. thanks the CERN Theoretical Physics Department for hospitality during the completion of this work. J.S. thanks the University of Florence and the Florence INFN division where a large fraction of this work was completed. $\mathrm{J}$. S. is grateful for the support by the $\mathrm{CP}^{3}$-Origins Centre. The $\mathrm{CP}^{3}$-Origins Centre is partially funded by the Danish National Research Foundation, Grant No. DNRF90. This work was supported by the ERC Grant NEO-NAT. We thank Martti Raidal and NICPB for allowing us to run our codes on their machines. The authors support equal opportunities.

\section{APPENDIX: NON-ABELIAN BOUND STATES}

Production cross sections and decay widths of two-body bound states due to perturbative non-Abelian gauge interactions have been given in [13], for bound states with low angular momentum $\ell$. Following the same notations, in Sec. A 2 we generalize the decay widths to any $\ell$. Although not needed in this work, in Sec. A 1 we also show the cross sections for formation of bound states with generic $\ell$. We consider emission of a single-vector $V^{a}$ in dipole approximation, such that the angular momenta of the initial and final states differ by $\Delta \ell= \pm 1$. We denote with $\alpha$ the nonAbelian gauge coupling, with $M_{a}$ the vector mass, and with $M$ the common mass of the two particles that form the bound state.

\section{Cross sections for bound state formation}

Production of a bound state with angular momentum $\ell$ proceeds from initial states with angular momentum $\ell \pm 1$ : $\left(\sigma_{\mathrm{bsf}}^{n \ell} v_{\mathrm{rel}}\right)_{a}=\left(\sigma_{\mathrm{bsf}}^{n} v_{\mathrm{rel}}\right)_{a}^{\ell-1 \rightarrow \ell}+\left(\sigma_{\mathrm{bsf}}^{n} v_{\mathrm{rel}}\right)_{a}^{\ell+1 \rightarrow \ell}$. The cross sections are 


$$
\begin{aligned}
\left(\sigma_{\mathrm{bsf}}^{n} v_{\mathrm{rel}}\right)_{a}^{\ell+1 \rightarrow \ell}= & \frac{8(\ell+1)}{2 \ell+3} \frac{\alpha k}{M^{2}}\left(1-\frac{k^{2}}{3 \omega^{2}}\right) \\
& \times\left|\int r^{2} d r \times R_{n \ell, j^{\prime} i^{\prime}}^{*}\left(\frac{1}{2}\left(T_{i^{\prime} i}^{a} \delta_{j j^{\prime}}-\bar{T}_{j^{\prime} j}^{a *} \delta_{i i^{\prime}}\right)\left(\partial_{r}+\frac{\ell+2}{r}\right)-i \frac{\alpha M}{2}\left(T_{i^{\prime} i}^{b} \bar{T}_{j^{\prime} j}^{c} f^{a b c}\right) e^{-M_{a} r}\right) R_{p, \ell+1, i j}\right|^{2}
\end{aligned}
$$

or, equivalently, integrating by parts

$$
\begin{aligned}
\left(\sigma_{\mathrm{bsf}}^{n} v_{\mathrm{rel}}\right)_{a}^{\ell+1 \rightarrow \ell}= & \frac{8(\ell+1)}{2 \ell+3} \frac{\alpha k}{M^{2}}\left(1-\frac{k^{2}}{3 \omega^{2}}\right) \\
& \times\left|\int r^{2} d r R_{p, \ell+1, i j}\left(\frac{1}{2}\left(T_{i^{\prime} i}^{a} \delta_{j j^{\prime}}-\bar{T}_{j^{\prime} j}^{a *} \delta_{i i^{\prime}}\right)\left(\partial_{r}-\frac{\ell}{r}\right)+i \frac{\alpha M}{2}\left(T_{i^{\prime} i}^{b} \bar{T}_{j^{\prime} j}^{c} f^{a b c}\right) e^{-M_{a} r}\right) R_{n \ell, j^{\prime} i^{\prime}}^{*}\right|^{2},
\end{aligned}
$$

where $R_{n \ell, i j}$ is the bound state wave function in the two-particle space $|i\rangle \otimes|j\rangle$ and $R_{p \ell, i j}$ is the wave function of the initial free state with relative momentum $p$ and angular momentum $\ell$. The other cross section is

$$
\begin{aligned}
\left(\sigma_{\mathrm{bsf}}^{n} v_{\mathrm{rel}}\right)_{a}^{\ell-1 \rightarrow \ell}= & \frac{8 \ell}{2 \ell-1} \frac{\alpha k}{M^{2}}\left(1-\frac{k^{2}}{3 \omega^{2}}\right) \\
& \times\left|\int r^{2} d r R_{n \ell, j^{\prime} i^{\prime}}^{*}\left(\frac{1}{2}\left(T_{i^{\prime} i}^{a} \delta_{j j^{\prime}}-\bar{T}_{j^{\prime} j}^{a *} \delta_{i i^{\prime}}\right)\left(\partial_{r}-\frac{\ell-1}{r}\right)-i \frac{\alpha M}{2}\left(T_{i^{\prime} i}^{b} \bar{T}_{j^{\prime} j}^{c} f^{a b c}\right) e^{-M_{a} r}\right) R_{p, \ell-1, i j}\right|^{2} .
\end{aligned}
$$

The formulas above simplify if the gauge group is unbroken, or at least if all vectors have a common mass. Then, a decomposition into irreducible representations allows one to reduce the cross sections to Abelian-like expressions:

$$
\begin{aligned}
& \left(\sigma_{\mathrm{bsf}}^{n} \nu_{\mathrm{rel}}\right)_{a}^{\ell+1 \rightarrow \ell}=\frac{8(\ell+1)}{2 \ell+3} \frac{\alpha k}{M^{2}}\left(1-\frac{k^{2}}{3 \omega^{2}}\right)\left|\int r^{2} d r R_{n \ell}^{*}\left(C_{\mathcal{J}}^{a M M^{\prime}}\left(\partial_{r}+\frac{\ell+2}{r}\right)+C_{\mathcal{T}}^{a M M^{\prime}} \frac{\alpha M}{2} e^{-M_{a} r}\right) R_{p, \ell+1}\right|^{2}, \\
& \left(\sigma_{\mathrm{bsf}}^{n} \nu_{\mathrm{rel}}\right)_{a}^{\ell-1 \rightarrow \ell}=\frac{8 \ell}{2 \ell-1} \frac{\alpha k}{M^{2}}\left(1-\frac{k^{2}}{3 \omega^{2}}\right)\left|\int r^{2} d r R_{n \ell}^{*}\left(C_{\mathcal{J}}^{a M M^{\prime}}\left(\partial_{r}+\frac{\ell-1}{r}\right)+C_{\mathcal{T}}^{a M M^{\prime}} \frac{\alpha M}{2} e^{-M_{a} r}\right) R_{p, \ell-1}\right|^{2},
\end{aligned}
$$

where the group-theory part has been factored out in the coefficients

$$
\begin{aligned}
& C_{\mathcal{J}}^{a M M^{\prime}} \equiv \frac{1}{2} \mathrm{CG}_{i j}^{M} \mathrm{CG}_{i^{\prime} j^{\prime}}^{M^{\prime} *}\left(T_{i^{\prime} i}^{a} \delta_{j j^{\prime}}+T_{j^{\prime} j}^{a *} \delta_{i i^{\prime}}\right)=\frac{1}{2} \operatorname{Tr}\left[\mathrm{CG}^{M^{\prime}}\left\{\mathrm{CG}^{M}, T^{a}\right\}\right] \\
& C_{\mathcal{T}}^{a M M^{\prime}} \equiv i \mathrm{CG}_{i j}^{M} \mathrm{CG}_{i^{\prime} j^{\prime}}^{M^{\prime} *}\left(T_{i^{\prime} i}^{b} T_{j j^{\prime}}^{c} f^{a b c}\right)=i \operatorname{Tr}\left[\mathrm{CG}^{M^{\prime}} T^{b} \mathrm{CG}^{M} T^{c}\right] f^{a b c}
\end{aligned}
$$

that hold separately for each initial channel $J$ and final channel $J^{\prime}$, using the notations of [13].

\section{Bound state decays}

The decay widths of a bound state through single-vector emission are obtained from the previous expressions substituting the free-particle final state wave function $R_{p \ell}$ with the wave function of the desired final bound states. Assuming again degenerate (or massless) vectors and a bound state in a representation $R$ with dimension $d_{R}$, we find

$$
\begin{aligned}
\Gamma\left(n, \ell \rightarrow n^{\prime}, \ell-1\right)= & \frac{1}{d_{R}} \frac{8 \ell}{(2 \ell+1)} \frac{\alpha k}{M^{2}}\left(1-\frac{k^{2}}{3 \omega^{2}}\right) \\
& \times \sum_{a M M^{\prime}}\left|\int r^{2} d r R_{n^{\prime}, \ell-1}^{*}\left(C_{\mathcal{J}}^{a M M^{\prime}}\left(\partial_{r}+\frac{\ell+1}{r}\right)+C_{\mathcal{T}}^{a M M^{\prime}} \frac{\alpha M}{2} e^{-M_{a} r}\right) R_{n \ell}\right|^{2}
\end{aligned}
$$

and 


$$
\begin{aligned}
\Gamma\left(n, \ell \rightarrow n^{\prime \prime}, \ell+1\right)= & \frac{1}{d_{R}} \frac{8(\ell+1)}{(2 \ell+1)} \frac{\alpha k}{M^{2}}\left(1-\frac{k^{2}}{3 \omega^{2}}\right) \\
& \times \sum_{a M M^{\prime}}\left|\int r^{2} d r R_{n^{\prime \prime} \ell+1}^{*}\left(C_{\mathcal{J}}^{a M M^{\prime}}\left(\partial_{r}-\frac{l}{r}\right)+C_{\mathcal{T}}^{a M M^{\prime}} \frac{\alpha M}{2} e^{-M_{a} r}\right) R_{n \ell}\right|^{2} .
\end{aligned}
$$

[1] L. Giusti, A. Romanino, and A. Strumia, Natural ranges of supersymmetric signals, Nucl. Phys. B550, 3 (1998).

[2] A. Strumia, The fine-tuning price of the early LHC, J. High Energy Phys. 04 (2011) 073.

[3] N. Arkani-Hamed and S. Dimopoulos, Supersymmetric unification without low energy supersymmetry and signatures for fine-tuning at the LHC, J. High Energy Phys. 06 (2005) 073.

[4] N. Arkani-Hamed, S. Dimopoulos, G. F. Giudice, and A. Romanino, Aspects of split supersymmetry, Nucl. Phys. B709, 3 (2004).

[5] G. F. Giudice and A. Strumia, Probing high-scale and split supersymmetry with Higgs mass measurements, Nucl. Phys. B858, 63 (2012).

[6] E. Bagnaschi, G. F. Giudice, P. Slavich, and A. Strumia, Higgs mass and unnatural supersymmetry, J. High Energy Phys. 09 (2014) 092.

[7] A. Arvanitaki, C. Davis, P. W. Graham, A. Pierce, and J. G. Wacker, Limits on split supersymmetry from gluino cosmology, Phys. Rev. D 72, 075011 (2005).

[8] V. De Luca, A. Mitridate, M. Redi, J. Smirnov, and A. Strumia, Colored Dark Matter, Phys. Rev. D 97, 115024 (2018).

[9] J. Kang, M. A. Luty, and S. Nasri, The Relic abundance of long-lived heavy colored particles, J. High Energy Phys. 09 (2008) 086.

[10] M. Toharia and J. D. Wells, Gluino decays with heavier scalar superpartners, J. High Energy Phys. 02 (2006) 015.

[11] P. Gambino, G. F. Giudice, and P. Slavich, Gluino decays in split supersymmetry, Nucl. Phys. B726, 35 (2005).

[12] A. De Simone, G. F. Giudice, and A. Strumia, Benchmarks for Dark Matter searches at the LHC, J. High Energy Phys. 06 (2014) 081.

[13] A. Mitridate, M. Redi, J. Smirnov, and A. Strumia, Cosmological implications of Dark Matter bound states, J. Cosmol. Astropart. Phys. 05 (2017) 006.

[14] K. Griest and M. Kamionkowski, Unitarity Limits on the Mass and Radius of Dark Matter Particles, Phys. Rev. Lett. 64, 615 (1990).

[15] M. Geller, S. Iwamoto, G. Lee, Y. Shadmi, and O. Telem, Dark quarkonium formation in the early universe, J. High Energy Phys. 06 (2018) 135.

[16] W. Fischler, $q-\bar{q}$ Potential in QCD, Nucl. Phys. B129, 157 (1977); Y. Schroder, The static potential in QCD to two loops, Phys. Lett. B 447, 321 (1999).

[17] P. Bicudo, The QCD string tension curve, the ferromagnetic magnetization, and the quark-antiquark confining potential at finite temperature, Phys. Rev. D 82, 034507 (2010);
P. Petreczky, Lattice QCD at non-zero temperature, J. Phys. G 39, 093002 (2012); S. Aoki et al., Review of lattice results concerning low-energy particle physics, Eur. Phys. J. C 77, 112 (2017); G. S. Bali, Casimir scaling of SU(3) static potentials, Phys. Rev. D 62, 114503 (2000).

[18] R. L. Hall, Simple eigenvalue formula for the Coulomb plus linear potential, Phys. Rev. D 30, 433 (1984).

[19] G. Bhanot and M.E. Peskin, Short distance analysis for heavy quark systems. 2. Applications, Nucl. Phys. B156, 391 (1979).

[20] A. Mitridate, M. Redi, J. Smirnov, and A. Strumia, Dark Matter as a weakly coupled dark baryon, J. High Energy Phys. 10 (2017) 210.

[21] M. Kawasaki, K. Kohri, and T. Moroi, Big-Bang nucleosynthesis and hadronic decay of long-lived massive particles, Phys. Rev. D 71, 083502 (2005).

[22] M. Kawasaki, K. Kohri, T. Moroi, and Y. Takaesu, Revisiting big-bang nucleosynthesis constraints on longlived decaying particles, Phys. Rev. D 97, 023502 (2018).

[23] M. Pospelov, Particle Physics Catalysis of Thermal Big Bang Nucleosynthesis, Phys. Rev. Lett. 98, 231301 (2007).

[24] M. Kusakabe, T. Kajino, T. Yoshida, and G. J. Mathews, Effect of long-lived strongly interacting relic particles on big bang nucleosynthesis, Phys. Rev. D 80, 103501 (2009).

[25] M. Kawasaki and T. Moroi, Electromagnetic cascade in the early universe and its application to the big bang nucleosynthesis, Astrophys. J. 452, 506 (1995).

[26] E. L. Wirght et al., Interpretation of the COBE FIRAS spectrum, Astrophys. J. 420, 450 (1994).

[27] W. Hu and J. Silk, Thermalization Constraints and Spectral Distortions for Massive Unstable Relic Particles, Phys. Rev. Lett. 70, 2661 (1993).

[28] FIRAS Collaboration, Measurement of the cosmic microwave background spectrum by the COBE FIRAS instrument, Astrophys. J. 420, 439 (1993).

[29] D. J. Fixsen, E. S. Cheng, J. M. Gales, J. C. Mather, R. A. Shafer, and E. L. Wright, The cosmic microwave background spectrum from the full COBE FIRAS data set, Astrophys. J. 473, 576 (1996).

[30] M. Cirelli, G. Corcella, A. Hektor, G. Hutsi, M. Kadastik, P. Panci, M. Raidal, F. Sala, and A. Strumia, PPPC 4 DM ID: A poor particle physicist cookbook for Dark Matter indirect detection, J. Cosmol. Astropart. Phys. 03 (2011) 051; Erratum, 10 (2012) E01.

[31] PIXIE Collaboration, The Primordial Inflation Explorer (PIXIE): A nulling polarimeter for cosmic microwave 
background observations, J. Cosmol. Astropart. Phys. 07 (2011) 025.

[32] T. R. Slatyer and C-L. Wu, General constraints on Dark Matter decay from the cosmic microwave background, Phys. Rev. D 95, 023010 (2017).

[33] V. Poulin, J. Lesgourgues, and P. D. Serpico, Cosmological constraints on exotic injection of electromagnetic energy, J. Cosmol. Astropart. Phys. 03 (2017) 043.

[34] J. D. Bowman, A. E. E. Rogers, R. A. Monsalve, T. J. Mozdzen, and N. Mahesh, An absorption profile centred at 78 megahertz in the sky-averaged spectrum, Nature (London) 555, 67 (2018).

[35] H. Liu and T. R. Slatyer, Implications of a 21-cm signal for Dark Matter annihilation and decay, Phys. Rev. D 98, 023501 (2018).

[36] S. Clark, B. Dutta, Y. Gao, Y.-Z. Ma, and L. E. Strigari, $21 \mathrm{~cm}$ limits on decaying Dark Matter and primordial black holes, Phys. Rev. D 98, 043006 (2018).

[37] A. Mitridate and A. Podo, Bounds on Dark Matter decay from $21 \mathrm{~cm}$ line, J. Cosmol. Astropart. Phys. 05 (2018) 069.

[38] T. Cohen, K. Murase, N. L. Rodd, B. R. Safdi, and Y. Soreq, $\gamma$-Ray Constraints on Decaying Dark Matter and Implications for IceCube, Phys. Rev. Lett. 119, 021102 (2017).

[39] M. Foster and C. Michael, Hadrons with a heavy color adjoint particle, Phys. Rev. D 59, 094509 (1999).
[40] G. R. Farrar, R. Mackeprang, D. Milstead, and J. P. Roberts, Limit on the mass of a long-lived or stable gluino, J. High Energy Phys. 02 (2011) 018.

[41] S. Polikanov, C. S. Sastri, G. Herrmann, K. Lutzenkirchen, M. Overbeck, N. Trautmann, A. Breskin, R. Chechik, and Z. Frankel, Search for supermassive nuclei in nature, Z. Phys. A 338, 357 (1991).

[42] ATLAS Collaboration, Reinterpretation of searches for supersymmetry in models with variable $R$-parity-violating coupling strength and long-lived $R$-hadrons, ATLASCONF-2018-003, https://atlas.web.cern.ch/Atlas/GROUPS/ PHYSICS/CONFNOTES/ATLAS-CONF-2018-003/.

[43] ATLAS Collaboration, Search for new phenomena in dijet events using $37 \mathrm{fb}^{-1}$ of $p p$ collision data collected at $\sqrt{s}=$ $13 \mathrm{TeV}$ with the ATLAS detector, Phys. Rev. D 96, 052004 (2017).

[44] M. Papucci, K. Sakurai, A. Weiler, and L. Zeune, Fastlim: A fast LHC limit calculator, Eur. Phys. J. C 74, 3163 (2014).

[45] J. Ellis, F. Luo, and K. A. Olive, Gluino coannihilation revisited, J. High Energy Phys. 09 (2015) 127.

[46] A. Delgado, G. F. Giudice, G. Isidori, M. Pierini, and A. Strumia, The light stop window, Eur. Phys. J. C 73, 2370 (2013).

[47] M. Pospelov and J. Pradler, Big bang nucleosynthesis as a probe of new physics, Annu. Rev. Nucl. Part. Sci. 60, 539 (2010). 